DIACRONIE

\section{Diacronie}

Studi di Storia Contemporanea

$N^{\circ} 13,1 \mid 2013$

Contrabbandieri, pirati e frontiere: per una storia delle pratiche informali nell'America Centrale (XVII-XXI

secolo)

\title{
La Période spéciale en temps de paix
}

Les différents visages de l'informalité cubaine

Janice Argaillot

\section{Q OpenEdition \\ Journals}

\section{Édition électronique}

URL : http://journals.openedition.org/diacronie/762

DOI : 10.4000/diacronie.762

ISSN : 2038-0925

\section{Éditeur}

Association culturelle Diacronie

\section{Référence électronique}

Janice Argaillot, «La Période spéciale en temps de paix », Diacronie [En ligne], № 13, 1 | 2013,

document 11, mis en ligne le 01 avril 2014, consulté le 01 mai 2019. URL : http://

journals.openedition.org/diacronie/762; DOI : 10.4000/diacronie.762 


\section{Diacronie}

N. 13 | 1|2013 Contrabbandieri, pirati e frontiere

\section{1/}

\section{La Période spéciale en temps de paix: les différents visages de l'informalité cubaine}

Janice ARGAILLOT *

Cet article offre une analyse des différentes formes d'informalité qui sont nées ou ont ressurgi avec la Période spéciale en temps de paix. Cette période consécutive à la chute de l'allié soviétique, caractérisée par des mesures économiques drastiques visant à préserver le système politico-social cubain, a effectivement entraîné des bouleversements dans différents domaines. De la sorte, l'informalité économique et le développement d'une économie parallèle ainsi que du marché noir doivent être observés au prisme de deux autres types d'informalité. Sur le plan politique d'abord, on ne peut que constater le mécontentement d'une certaine partie de la population, à laquelle les nouvelles technologies offrent une tribune internationale. Sur un plan humain ensuite, il convient de souligner que la Période spéciale a provoqué une mutation des relations sociales, tout en s'imposant comme une source d'inspiration pour les artistes.

Notre travail offre donc un panorama des différents visages de "l'informalité" cubaine actuelle, et s'attache à en souligner les aspects négatifs comme positifs.

vec la disparition du camp socialiste s'est ouvert à Cuba ce que l'on a
appelé « la Période spéciale en temps de paix ». Cette période, dont lîle
n'est toujours pas officiellement sortie à ce jour, s'est caractérisée par des
mesures drastiques visant à préserver l'économie du pays (remise en place du carnet de rationnement, coupures d'électricité planifiées, etc.). En conséquence, les Cubains ont dû mettre en place les stratégies les plus variées afin de conserver des 
conditions de vie décentes, et se sont mis à conjuguer le verbe « resolver » à tous les temps.

Nous nous proposons donc dans un premier temps d'analyser l'informalité économique née de cette période particulièrement difficile. En effet, le marché noir s'est imposé comme une planche de salut, et l'arrivée d'un flot conséquent de touristes a signifié la mise en place d'une économie parallèle qu'il convient d'analyser avec attention, dans la mesure où elle a conduit à la création d'une société à deux vitesses. Dans un deuxième temps, nous souhaitons mettre en avant l'informalité politique, fille du désenchantement provoqué par la crise économique. Il est clair que la Période spéciale a offert un nouvel espace à la « dissidence », mais elle a également provoqué un détachement vis-à-vis de la Révolution chez de nombreux Cubains, qui, sans s'opposer à elle, ne s'y sentent plus clairement identifiés. Dès lors, c'est une division profonde des acteurs de la société qui se met en place.

Enfin, nous aborderons l'informalité sociale et culturelle provoquée par la crise subie l'île. En effet, les problèmes économiques et politiques ont débouchés sur de nouvelles pratiques culturelles, liées à la marginalisation ou à l'auto-marginalisation de tout un pan de la société, et peuvent ainsi être perçus comme les moteurs de nouveaux courants artistiques et identitaires.

\section{Informalité économique}

Il faut avant tout rappeler que l'effondrement du bloc soviétique constitua un véritable raz-de-marée pour l'économie cubaine, auquel s'ajoutait le renforcement du blocus états-unien. La population de l'île se vit ainsi dans l'obligation de trouver des solutions pour survivre, et on observe que le début des années 1990 est synonyme d'un développement conséquent du marché noir. En parallèle, le verbe «resolver» est devenu une sorte de leitmotiv, qui, conjugué à tous les temps, ponctue le quotidien des Cubains : "Resolver means to resolve, but in Cuba resolver has become synonymous with survival and is ingrained in daily conversation: ¿Cómo estás? Aquí, resolviendo. Life in Cuba is a struggle. No es fácil, it's not easy, is the common refrain. Everyday items that we take for granted like food, fuel, medicine and clothing can be difficult to obtain. The simplest errand can become an arduous challenge» ${ }^{1}$.

La vie semble donc être une succession de luttes, et la crise économique a en quelque sorte dérobé leur futur aux Cubains, les obligeants à vivre au jour le jour. Le

\footnotetext{
${ }^{1}$ NEYRA, Edward J., Cuba Lost and Found, Cincinnati, Clerisy Press, 2010, p. 218.
} 
gouvernement de l'île a tenté de trouver des solutions aux difficultés économiques. En effet, deux monnaies ont actuellement cours à Cuba : le CUC, indexé sur la valeur du dollar états-unien (créé par l'État cubain dans le but de ne plus laisser libre court au billet vert, symbole de la puissance économique de l'ennemi héréditaire), et le peso cubain, la moneda nacional. «Entre 1992 et 1994, le dollar devient, à l’instar du peso cubain, monnaie officielle $[. ..] »^{2}$. Le CUC (peso convertible) fut quant à lui adopté comme monnaie officielle le 8 novembre 2004, et il constitue le seul moyen pour les Cubains de racheter certains produits (lait, produits d'hygiène corporelle...) lorsque ceux qui leur ont été attribués par le biais du carnet de rationnement sont épuisés.

Ainsi, l'interdiction de posséder des dollars dans l'île, puis du CUC, n'a pas résolu les problèmes que son autorisation avait entraîné : «Since 1991, the Cuban economy has been in deep crisis, and the government is effectively brankrupt. [...] The informal economy has grown since the economic crisis worsened. For example, many professionals also work in the informal sector. Cuba is moving toward a dual economy defined by dollars : those who have them and those who do not»3

La «dualisation » de l'économie engendre en outre de nombreux problèmes. La création d'une société à deux vitesses au sein de bastion du communisme puis du socialisme latino-américain peut surprendre - «l'idéologie cesse, dès 1992, d'être « marxiste-léniniste » pour devenir celle de la « Nation cubaine »4 -, mais apparaît néanmoins comme une évidence : «[...] l'égalité sociale, si chère au communisme, est remise en cause »5. Il faut dire que les deux sociétés qui ont surgi à Cuba, l'une formelle et l'autre informelle, ne sont pas strictement parallèles, puisqu'elles s'entremêlent plus qu'elles ne s'entrechoquent. En effet, les travailleurs informels cubains ne sont pas nécessairement pauvres et marginaux, ils ne sont pas obligatoirement au ban de la société. Tout au contraire, beaucoup de ressortissants de l'île ont une activité formelle, qu'ils sont obligés de compléter par une ou des activités informelles. Pour les Cubains, il s'agit de pallier les problèmes ; l'économie informelle agit donc en complémentarité du système « officiel ».

Néanmoins, on observe bien une division de la société, qui est une résultante de la mise en place du double système monétaire, dans la mesure où les Cubains travaillant au contact des touristes peuvent être payés en CUC, et où les différences de salaire

${ }^{2}$ MICHAUT, Maxime, Cuba: l'encadrement idéologique et social face à la politique de déstabilisation des États-Unis, Paris, Editions L’Harmattan, 2006, p. 37.

3 MICKELSON, Roslyn A. (ed.), Children on the Streets of the Americas: Globalization, Homelessness and Education in the United States, Brazil, and Cuba, London-New York, Routledge, 2000, p. 21.

4 MICHAUT, Maxime, op. cit., p. 42.

5 Ibidem, p. 41. 
(pourboires compris) et de conditions de vie entre les Cubains ayant une activité professionnelle liée au tourisme et les autres sont absolument énormes. On observe d'ailleurs que les reconversions sont désormais monnaie courante, puisque les chauffeurs de taxi gagnent plus que les médecins, et que s'ouvrent de nombreux paladares, sorte de petites auberges tenues par des particuliers au sein même de leur maison, ne pouvant accueillir plus de vingt convives, que fréquentent les touristes mais également depuis peu quelques Cubains. Ainsi, tout est mis en place pour que les Cubains fréquentant les touristes se convertissent en une sorte de petite bourgeoisie au sein de la société révolutionnaire.

D'ailleurs, les cuentapropistas, personnes qui travaillent pour leur propre compte, prennent de plus en plus de poids : « [...] en 1995, on dénombre officiellement près de 200.000 cuentapropistas, signe d'une réelle volonté d'émancipation économique ${ }^{6}$. Ces travailleurs indépendants peuvent plus facilement que les autres cacher les revenus réels de leur travail.

La double monnaie cubaine est donc liée au tourisme. Cette activité est à son tour en lien avec la prostitution (jineterismo) féminine et masculine, et avec la criminalité : «Ainsi depuis quelques années, la prostitution que l'on croyait disparue commence-telle à réapparaître en force dans certaines grandes villes portuaires, à La Havane en particulier. Il ne s'agit plus d'un phénomène social, tel qu'il existait encore dans les premières années de la Révolution. Il résulte plutôt de la politique marquée de Cuba en faveur du tourisme international» 7 . Pour le dire autrement, les autorités cubaines ont vu leur salut dans l'ouverture de l'île au tourisme. Cette ouverture n'est pas sans conséquence, et si on peut souligner le dynamisme qu'elle a insufflé à l'artisanat cubain, il convient également de mettre en avant le développement d'un tourisme sexuel.

En outre, l'arrivée de touristes occidentaux bardés du matériel technologique dernier cri provoque de nombreuses interrogations quant au système cubain, mais également une envie qui se ressent dans l'augmentation du nombre de vols à l'arrachée dans les quartiers touristiques : "Le tourisme étranger, un mal nécessaire aux dires des dirigeants, est un élément dont l'incidence négative est la plus visible sur les jeunes. Le touriste qui arrive à Cuba, issu en majorité des secteurs moyens des sociétés capitalistes, agit à Cuba en représentant direct de la société de consommation ${ }^{8}$.

Finalement, les bouleversements induits par la Période spéciale sont un facteur de stress pour les habitants de l'île, et l'essor du tourisme a provoqué l'éclosion d'une

\footnotetext{
${ }^{6}$ Ibidem.

7 GAY-SYLVESTRE, Dominique, ESTRADE, Paul, Être femme à Cuba: des premières militantes féministes aux militantes révolutionnaires, Paris, Editions L’Harmattan, 2006, pp. 234-235.

8 Ibidem.
} 
économie parallèle que l'on retrouve dans de nombreux pays en voie de développement: "The introduction of a new commercialism that commenced during the Special period in Peacetime has created fresh problems for Cuban families already stressed by long-term economic crisis. The explosive growth of the tourist industry [...] created markets for illicit street life, including prostitution among young women and men. There has also been an increase in the number of children besieging tourists by either begging or hawking merchandise or services in the informal sector» 9 .

Les autorités ont conscience du phénomène, et les lois promulguées punissent sévèrement les Cubains qui s'adonnent au marché noir, à la contrebande, à la prostitution... Il s'agit là d'un crime économique mais également politique, dans la mesure où ces activités sont perçues comme contre-révolutionnaires. Néanmoins, ces mêmes autorités ne peuvent que faire preuve de tolérance, ou du moins d'indulgence, au vu de l'ampleur de la crise. La totalité de la société étant touchée, les policiers n'ont d'autre choix que de fermer les yeux sur certains agissements, leurs propres familles ayant parfois recours au « système $\mathrm{D}$ ».

Ainsi, malgré les risques encourus, beaucoup de Cubains continuent à faire vivre l'économie informelle ou parallèle. Celle-ci a d'ailleurs pris une importance considérable, et se pose en pilier de l'économie générale cubaine : «Pese al riesgo de fuertes multas y penas de prisión prolongadas y a la omnipresencia del Estado, la economía informal cubana ha florecido y en el año 2000 representó, según una estimación reciente, hasta 40\% del PIB. Existen establecimientos clandestinos donde se fabrican y reparan motores para bombas de agua y refrigeradores, se elaboran bebidas sin alcohol y cerveza y se producen cigarros para exportación. La construcción de la vivienda propia y especialmente su reparación se realizan cada vez más a través de la economía informal $»^{10}$. D’autres estimations montrent bien que toute la population a recours, à des degrés différents, à l'économie informelle: «Según estimaciones extraoficiales en la economía informal circulan alrededor de 2 mil millones de pesos anuales, aproximadamente un 20\% de los gastos totales en que incurre la población. De acuerdo con los mismos estimados más del 70\% de los núcleos familiares incursionan en el sector informal como compradores ${ }^{11}$. On observe que différents secteurs sont touchés par l'économie informelle, même si celui des services semble être le pivot de

\footnotetext{
9 MICKELSON, Roslyn A., op. cit., p. 27.

${ }^{10}$ PORTES, Alejandro, HALLER, William, La economía informal, Santiago de Chile, Naciones Unidas, CEPAL, División de Desarrollo Social, 2004, p. 18.

${ }^{11}$ GONZÁLEZ NÚÑEZ, Gerardo, Cuba y el mercado mundial, in RODRÍGUEZ BERUFF, Jorge (compilador), Cuba en crisis : perspectivas económicas y políticas, San Juan, Editorial de la Universidad de Puerto Rico, 2005, p. 53.
} 
l'économie parallèle. A titre d'exemple, on peut signaler que de nombreux cubains vendent clandestinement des appareils électroménagers ${ }^{12}$.

Il convient donc de noter les particularités du système cubain, qui bien qu'en crise, continue à prôner la justice sociale à travers l'éducation et de la santé :

A partir de 1990, [la Période spéciale] est le nom qui a été donné à une étape exceptionnellement critique du processus révolutionnaire cubain. Elle début lorsque le dénommé bloc socialiste s'effondre et que Cuba perd ses principaux partenaires économiques. A cela s'ajoute le renforcement de l'embargo commercial des États-Unis. Au cours de cette période, le gouvernement a adopté une stratégie de concentration des ressources et de restriction des niveaux de consommation de la population et de l'activité économique. Cependant, malgré la crise économique, pas une seule école ni une maison de vieux, ni un hôpital n'ont fermé leurs portes. La protection sociale est restée en vigueur, bien qu'avec des déficiences compréhensibles ${ }^{13}$.

Ainsi, la «pauvreté » et l'informalité cubaine seraient finalement bien différentes de celles que l'on retrouve sur le sous-continent latino-américain, l'État permettant malgré tout le maintien d'une certaine qualité de soins, l'accès à l'éducation, etc. ${ }^{14}$. Cela étant, l'économie informelle cubaine ne doit pas être uniquement perçue -même si cela semble antithétique- dans sa dimension économique, puisqu'on a souvent dit d'elle qu'elle pouvait permettre une « transition politique » dans l'île.

\section{Informalité politique}

Si certains cubains ne peuvent clairement être identifiés comme «contrerévolutionnaires ", ils ne sont à l'inverse pas -ou plus- tout à fait convaincus par leur système politique. Et l'une des caractéristiques de la Révolution cubaine est précisément l'attachement de la population à sa Révolution ainsi qu'à certaines de ses « grandes figures ». Le système révolutionnaire n'est effectivement pas, contrairement à ce que l'on a pu dire et écrire, une "simple dictature ». Le manque de liberté d'expression n'est sans doute pas réfutable, mais il faut également mettre en avant le

12 PORTES, Alejandro, HALLER, William, op. cit., pp. 18-19.

13 CALVO OSPINA, Hernando, DECLERCQ, Katlijn, Dissidents ou mercenaires? Des EtatsUnis à l'Europe pour briser Cuba, Bruxelles, Editions Aden, 1998, p. 207.

14 MICKELSON, Roslyn A., op. cit., p. 21. 
lien entre les Cubains et « leur » Révolution. La fracture entre le peuple cubain et les autorités serait comparable à une rupture amoureuse.

Le nerf de la question semble encore une fois être l'argent :

Un [...] motif d'ailleurs lié à celui de la faim et source d'inégalité dans la société révolutionnaire [...] est celui du pouvoir du dollar, autrefois considéré comme un symbole de l'impérialisme yanqui. Objet de convoitise, il est au centre d'une hiérarchisation tragique pour le pays entre ceux qui en détiennent et les autres, et à l'origine d'une décomposition des mœurs et de situation absurde pour la population. Eliseo Alberto constate, dans Informe contra mí mismo, qu'il est un enjeu majeur dans le dialogue qu'engagent les autorités cubaines avec les dissidents dans les années 1995 car c'est le dollar qui détermine quelle catégorie d'opposants mérite d'être écoutée et considérée : [...] los de afuera tienen dólares, los de adentro dolores ${ }^{15}$.

La crise est un motif de contestation, et offre dans le même temps une tribune rêvée aux opposants.

Si l'État cubain reconnaît la liberté de religion et d'association, exprimer son opposition à la politique mise en place par les autorités n'est pas toléré : « [...] toute structure un minimum organisée est sous emprise directe ou indirecte de l'État, à commencer par les canaux d'information $»^{16}$. Par ailleurs, on procède à une mise à la marge de ceux qui ne sont pas contre la Révolution, mais ne s'engagent pas non plus pour elle. L'informalité prend ici tout son sens, puisque ne pas prendre le chemin officiel signifie une possibilité d'exprimer une voix contraire à celle du gouvernement, mais constitue également un risque de se voir exclu de la société. En outre,

le développement du tourisme et des relations diplomatiques de Cuba tout au long des années 1990 ont offert les moyens à la dissidence de se faire connaître à l'étranger. Avec l'ouverture au monde décrétée par le régime, l'opposition se médiatise, notamment grâce à l'ampleur que prend le journalisme indépendant à Cuba. Le développement des nouvelles technologies aidant, des agences de presse illégales telles que Cubanet, Cuba Free Press, Carta de Cuba ou encore Nueva

15 LUCIEN, Renée Clémentine, Résistance et cubanité. Trois écrivains nés avec la Révolution cubaine - Eliseo Alberto - Leonardo Padura - Zoé Valdés, Paris, Editions L’Harmattan, 2006, p. 129.

${ }^{16}$ MICHAUT, Maxime, op. cit., p. 33. 
Prensa de Cuba font leur apparition dès 1995, permettant à l'information de circuler à l'intérieur mais surtout vers l'extérieur de l'île ${ }^{17}$.

Il s'agit d'une crise globale, puisque

[...] même les plus fervents partisans du régime peuvent se mettre à douter. Cuba n'est plus le monde clos et imprenable qu'il a pu être pendant les premières années de la Révolution. Le cap des deux millions de touristes franchi en 2004 rend impossible la surveillance de toutes les interactions entre les visiteurs et la population. Le tourisme semble être devenu plus que jamais le « cheval de Troie » cubain des critiques internationales ${ }^{18}$.

En somme, les nouvelles connexions de Cuba avec le monde extérieur favorise la corruption mais également la contestation d'un régime que l'on perçoit comme étant de plus en plus archaïque.

La Période spéciale s'impose dès lors comme « une remise en cause de l'identité du régime » qui, ajoutée à l'ouverture de l'île au tourisme, a « offert aux opposants des moyens jusque là inexistants de déstabiliser les institutions ${ }^{19}$. Il ne s'agit plus uniquement de s'opposer, d'exprimer un avis contraire, de critiquer, mais bien de tenter de « déstabiliser » le régime en place. En quelques mots, il faut dire que certains voyaient dans la Période spéciale la fin du « régime castriste », et que si l'on ne peut que constater qu'il n'en fut rien, la Période spéciale a redynamisé la voix de la contestation, en lui offrant de nouveaux moyens d'expression.

Ainsi, la dureté de la crise subie et l'ouverture progressive aux nouvelles techniques de communication se sont conjuguées pour ouvrir une tribune à tous ceux qui souhaitent voir s'instaurer des changements politiques dans l'île. A titre d'exemple, soulignons que la blogueuse Yoani Sánchez s'est faite connaître grâce à internet :

Yoani Sanchez is a true rebel [...]. The tongue-in-cheek harsh critics on her popular blog called Generación Y hit the Castro brothers like a brick. [...] In 2002 she emigrated to Switzerland but only two years later, homesick perhaps, decided to go back regardless of the opposite advice given by friends and family. Once back, computer science seemed to be a better option and way of expression than traditional writing ${ }^{20}$.

${ }^{17}$ Ibidem, p. 44.

18 Ibidem, p. 46.

19 Ibidem, p. 47.

${ }^{20}$ LUPI, Gordiano, Mi Cuba, Milano, Mediane, 2009, p. 265. 
L'audience de Yoani Sánchez est néanmoins principalement étrangère à Cuba, ce qui entraîne de nombreuses questions quant à la répercussion de son action. D’ailleurs, on constate que la dissidence est soutenue voire promue depuis l'extérieur: l'importance des États-Unis dans la création et le développement d'une dissidence ou à tout le moins d'une informalité politique est indéniable, et s'explique aisément, mais on observe également le soutien d'organisations non cubaines, tel Reporters sans frontières, aux mouvements dissidents.

Malgré tout, même si « l'État cubain n'est plus le seul employeur de l'île » et s'il a renoncé «au monopole du commerce extérieur ${ }^{21}$, il conserve une importance non négligeable dans le vie des Cubains :

L'État cubain a perdu de nombreux monopoles depuis la chute du communisme soviétique et la mise en place de la "période spéciale », mais il reste dans de très nombreux domaines l'Alpha et l'Oméga de tout projet ou initiative citoyenne. Quiconque cherche un logement, du travail, une place à l'université ou le droit de sortir du territoire 22 [...] doit s'adresser au gouvernement afin que celui-ci puisse contrôler et rétribuer de façon adéquate sa fibre révolutionnaire. Quiconque est affublé du qualificatif « contre-révolutionnaire » dans son dossier personnel n’a plus aucun espoir de développement personnel [...] ${ }^{23}$.

En conséquence, on peut se demander quel est le poids d'une informalité politique au sein d'un système qui apparaît comme fermé, et notamment concernant la vie sociale et culturelle.

\section{Informalité sociale et culturelle}

Il convient de souligner les changements dans les codes sociaux et moraux observés à Cuba depuis le début de la Période spéciale. Si la généralisation ne permet sans doute pas une étude scientifique réelle, on peut ainsi noter la banalisation de certains comportements : "Presque tout le monde vole, détourne pour survivre et alimenter le marché noir $[\ldots ..] »^{24}$. En parallèle, la corruption et les scandales financiers « laissent du régime une image bien ternie $»^{25}$.

\footnotetext{
${ }^{21}$ MICHAUT, Maxime, op. cit., pp. 41-42.

${ }^{22}$ Les lois ont évolué dans ce domaine depuis l'arrivée au pouvoir de Raúl Castro.

23 MICHAUT, Maxime, op. cit., p. 33.

24 Ibidem, p. 41.

25 Ibidem, p. 42.
} 
En outre, toutes les relations sociales sont perturbées. La pyramide sociale liée à l'économie formelle, déjà entamée par la politique révolutionnaire cubaine, n'a plus aucun sens. En effet, les Cubains ne se préoccupent plus de leur position dans un système formel ${ }^{26}$ qui ne leur apporte finalement rien :

A Cuba, on appelle ce phénomène la «pyramide inversée ». Une large part de la population la moins éduquée gagne plus d'argent que les traditionnelles élites. [...] Ce phénomène a lieu dès lors qu'il existe une disparité trop importante entre les salaires des personnes travaillant dans le secteur du tourisme et le reste de la population locale ${ }^{27}$.

Il faut alors se demander si l'on peut parler d'une évolution ou d'un bouleversement de la culture ainsi que des pratiques culturelles.

Rappelons à ce sujet que l'ouverture du pays à certaines mesures « capitalistes » induit une mutation des valeurs sociales : «Certaines valeurs d'égalité et de solidarité commencent à s'éroder, tandis que d'autres, relatives à l'initiative et à la réussite personnelles, gagnent du terrain ${ }^{28}$. C'est une mutation du système de pensée sur lequel était fondée la société que l'on observe ici.

En parallèle, on peut s'interroger sur l'identité des « acteurs informels » ou « de l’informalité ». S'agissant d'une crise globale, la totalité de la société est impliquée dans l'informalité. Néanmoins, on constate que les milieux intellectuels jouent un rôle prépondérant dans la diffusion d'une nouvelle culture et dans l'exploration d'une identité remise en cause par la crise. En effet, le chancellement du système politique induit de nombreuses questions pour tous ceux qui avaient fait de la Révolution le centre de l'identité culturelle cubaine.

Ainsi, nous pouvons dire que l'informalité économique et culturelle a permis dans une certaine mesure de rendre plus souples les débats autour de la culture. Le mot d'ordre semble être celui de l'intégration, et l'on tend à écarter les codes trop strictes ne permettant pas la véritable expression des sentiments des artistes ou auteurs Cubains : «Le projet culturel reconnaît désormais, incorpore et même promeut la diversité des

${ }^{26}$ DOMÍNGUEZ, María Isabel, La juventud cubana en una época de crisis y reestructuración, in BENÍTEZ, Jorge (compilador), Cuba hoy: desafios de fin de siglo, Santiago de Chile, Lom Ediciones, 1995, p. 94.

27 LAMIC, Jean-Pierre, Tourisme durable: utopie ou réalité?: Comment identifier les voyageurs et voyagistes éco-responsables ?, Paris, L'Harmattan, 2008, p. 74.

${ }_{28}$ LITZLER, Damien, Rythme musical, rythme identitaire, rythme social ou comment la Timba cadence les tensions à Cuba depuis les années 1990, in LE QUÉAU, Pierre (sous la direction de), 20 ans de sociologie de l'art : bilan et perspectives - tome 1, Paris, L’Harmattan, 2007, p. 40. 
formes et des contenus. Devenu plus intégrateur, il proclame ne vouloir laisser personne à l'écart» ${ }^{29}$.

Il y a donc une évolution de l'image de l'artiste ou de l'intellectuel dont la production n'est pas jugée strictement révolutionnaire ou en faveur de la Révolution :

Les intellectuels critiques ne sont plus des moutons noirs ou des francs-tireurs. La critique du régime, la contestation s'exprime aujourd'hui dans des œuvres exposées ou éditées dans lîle. La mise en cause la plus agressive n'a cependant pas encore tout à fait droit de cité ${ }^{30}$.

Même si l'on ne peut pas parler de liberté totale, il faut reconnaître que la Période spéciale constitue un tournant historique sans précédent depuis 1959, en ce qui concerne l'assouplissement des codes de création artistiques.

Cette évolution est à la fois le reflet et la conséquence directe de la crise globale subie par l'île. En effet, l'informalité économico-politique semble entraîner une liberté de ton, et la critique du système (certains diront du « régime ») semble plus aisée : « Si jusqu'aux années 1980 la critique restait aimablement culturelle et sociale, elle est devenue plus politique, plus sociétale, très caustique voire démolisseuse chez les jeunes intellectuels ${ }^{31}$.

Les artistes et créateurs culturels deviennent alors à la fois un rempart et un palliatif :

La presse ne jouant pas son rôle, les écrivains, les peintres mettent l'accent sur les plaies du présent, sur ses côtés obscurs. [...] Leurs œuvres en disent plus sur la réalité que nombre d'essais, d'articles. Le thème des balseros (boat people) est devenu récurrent chez des peintres comme Douglas Pérez Castro, Kcho, Sandra Ramos Lorenzo ; d'autres comme Carmen María Cabrera et José Angel Toirac travaillent sur le délabrement de La Havane, la décomposition sociale, etc ${ }^{2}$.

Dès lors, l'informalité devient une source de création, d'inspiration. Ainsi, les Arts et la politique s'entremêlent-ils dans la dénonciation des faiblesses du système :

29 ORTIZ, Jean, La période spéciale : une chance pour la culture cubaine, in LERAT, Christian (sous la direction de), Le monde caraïbe: défis et dynamiques, t. II, Pessac, Maison des Sciences de l'Homme d'Aquitaine, 2005, p. 170.

3o Ibidem.

${ }^{31}$ Ibidem, pp. 170-171.

32 Ibidem, p. 171. 
Une littérature de la gueule de bois, de la dérision, désenchantée, d'un pessimisme foncier, très réaliste, parfois violente, aborde aujourd'hui des thèmes hier tabous, refoulés ou censurés, tels que l'homosexualité, la discrimination religieuse, la marginalité, les déchirures de la guerre d'Angola, la débâcle du socialisme, la double morale, les nouveaux riches, la corruption en col blanc, la prostitution, la drogue, l'avenir incertain, la douleur de l'exil, etc. Depuis une quinzaine d'années, une pléiade de nouveaux écrivains participent d'un véritable «boom du romain cubain »: Leonardo Padura, [...] Abilio Estévez, Julio Travieso, Jorge Luis Hernández, Alexis Díaz Pimienta, Ronaldo Menéndez, Mylene Fernández [...]33.

Le vent de liberté qui paraît inspiré par l'informalité déconcerte sans doute : « La liberté de ton et de contenu des revues culturelles et universitaires étonne. Elles témoignent de

la rénovation de la pensée culturelle à Cuba [...]. Tout au long de la "période spéciale "sont nées (ou ont pris un nouveau départ) davantage de revues qui traduisent comme jamais auparavant des aspects de la pensée culturelle, sociale et politique: La Gaceta de Cuba, Temas, Revolución y Cultura, Catauro, Opus Habana, Contracorriente $[\ldots] 34$.

On observe un effet domino de l'économie informelle que la Période spéciale rend particulier. Les groupes sociaux et culturels nés de l'économie informelle sont effectivement atypiques dans ce contexte spécial :

Crecimiento de los grupos vinculados a la economía sumergida que implica, a su vez, incremento de los grupos de conducta desviada. El surgimiento y desarrollo de esta estructura social informal, correspondiente a una economía informal, resulta en la actualidad altamente compleja, pues no se trata de una estructura marginal a la sociedad, de una estructura residual, como era en los años 80, sino una estructura paralela a la formal, en ocasiones fuerte, porque de la inserción de los individuos en ella va a depender el nivel real de sus ingresos, su nivel de vida e incluso su estilo de vida35.

On constate en parallèle un repli vers, ou une redynamisation des cultes afrocubains telle la santería, ce qui montre bien la perte de certains repères, et la tentative

\author{
33 Ibidem. \\ 34 Ibidem. \\ 35 DOMÍNGUEZ, María Isabel, op. cit., p. 91.
}


de retrouver un socle commun à une identité désormais chancelante. Celle-ci est construite sur l'expérience quotidienne de la difficulté, et tend en conséquence à apporter des réponses que l'on veut immédiates à des problèmes dans lesquels la population se sent enlisée depuis trop longtemps :

[...] à l'opposition messianiste du castrisme et du catholicisme, systèmes structurés et fondés sur des fins dernières, répondrait une opposition de l'immédiateté, de la survie, qui serait celle de la période spéciale et de la santería, non systèmes visant à la résolution des problèmes au jour le jour ${ }^{36}$.

De la même façon, « [1]'explosion » de la timba à Cuba coïncide avec le début de la Période spéciale en temps de paix $\gg 37$. A travers la redécouverte de nouvelles pratiques culturelles, la population cherche un salut qui semble finalement lointain, et ces nouvelles pratiques deviennent un facteur de cohésion sociale : « La timba maintient dans une certaine mesure un ordre social mais aussi culturel dans le chaos économique ambiant $[\ldots] » 3^{8}$. En effet, si les valeurs sociales s'écroulent, si le système politique est remis en cause, il semble de plus en plus important de retrouver une certaine union, voire un trait d'union permettant de se sentir liés aux autres.

En somme, face au «chaos» et à l'impossibilité apparente de remédier à la situation, le lien culturel et la création artistiques s'imposent comme des planches de salut : « Les Cubains plongent dans le doute et l'exaspération face à une situation qui voit s'opposer l'idéal/l'idéel au réel, l'officiel à l'officieux, le conçu et le vécu. Le paradoxe et la contradiction rythment complètement la société cubaine depuis le début de la période spéciale et ce mouvement dialectique engendre de la nouveauté dans les structures. Il se traduit culturellement par des expressions innovantes en même temps qu'un certain repli sur la tradition. Rêves et frustrations, nostalgies et critiques, amour et haine sont évoqués à travers la diversité d'artistes de cette génération qui affrontent les problématiques de leur pays et les transformations qu'il a connues. On est étonné du sérieux et de l'importance vitale que revêt la pratique artistique pour ces témoins et acteurs d'une révolution en crise. «Elle me sauve la vie», déclare l'écrivain Angel Santiesteban à propos de la littérature : «S’il me manque la musique, c'est comme s’il me manquant la vie » insiste José Luis Cortés en entretien»39.

36 LÉTRILLIART, Philippe, Cuba, l'Église et la révolution: approche d'une concurrence conflictuelle, Paris, Editions L'Harmattan, 2005, p. 115.

37 LITZLER, Damien, op. cit., p. 39.

${ }^{38}$ Ibidem, p. 45.

39 Ibidem, p. 40. 


\section{Conclusion}

En conclusion, il est important de souligner que la Période spéciale fut, et est encore peut-être une crise globale, touchant toutes les sphères de la société, et mettant en scène de nombreux acteurs: "Véritable tremplin pour les opposants au régime castriste, la récession des "années noires" aura eu des effets aussi bien économiques, politiques, que sociaux ${ }^{40}$. Ainsi, les difficultés économiques et les stratégies mises en place par la population ont débouché sur la création d'une nouvelle société, qui, déstabilisée par la division dont elle est victime, cherche à recréer des liens grâce à la culture et à l'identité, liens qui peuvent s'établir dans la contestation du système politique.

L’impact de cette période définitivement spéciale est donc multiple, et il n'est pas dit que nous disposions actuellement du recul nécessaire pour en prendre toute la mesure. Néanmoins, on peut souligner que la Période spéciale induit une remise en cause du système politique, économique et social dans lequel ont été élevés la plupart des Cubains vivant sur l'île. En effet, les générations ayant connu la révolution en armes (1956-1959) ou la dictature de Fulgencio Batista ne sont désormais plus majoritaires sur le plan démographique. Conjuguée aux changements introduits depuis plusieurs années maintenant par Raúl Castro, cette nouvelle étape constitue sans doute un tournant historique pour l'île et ses habitants.

40MICHAUT, Maxime, op. cit., p. 42. 


\section{* L'auteur}

Janice Argaillot est docteur en études hispanophones (spécialité civilisation latino-américaine) et enseignante contractuelle de l'Université de Cergy-Pontoise (UFR Langues et Études Internationales).

URL: < http://www.studistorici.com/progett/autori/\#Argaillot >

\section{Per citare questo articolo:}

ARGAILLOT, Janice, «La Période spéciale en temps de paix: les différents visages de l'informalité cubaine», Diacronie. Studi di Storia Contemporanea: Contrabbandieri, pirati e frontiere: per una storia delle pratiche informali nell'America Centrale (XVII-XXI secolo), 29/04/2013,

URL:<http://www.studistorici.com/2013/04/29/argaillot_numero_13/ >

Diacronie Studi di Storia Contemporanea $\vartheta$ www.diacronie.it

Risorsa digitale indipendente a carattere storiografico. Uscita trimestrale.

redazione.diacronie@hotmail.it

Comitato di redazione: Marco Abram - Jacopo Bassi - Luca Bufarale - Alessandro Cattunar - Elisa Grandi - Deborah Paci - Fausto Pietrancosta - Matteo Tomasoni - Luca Zuccolo

Diritti: gli articoli di Diacronie. Studi di Storia Contemporanea sono pubblicati sotto licenza Creative Commons 2.5. Possono essere riprodotti a patto di non modificarne i contenuti e di non usarli per fini commerciali. La citazione di estratti è comunque sempre autorizzata, nei limiti previsti dalla legge. 